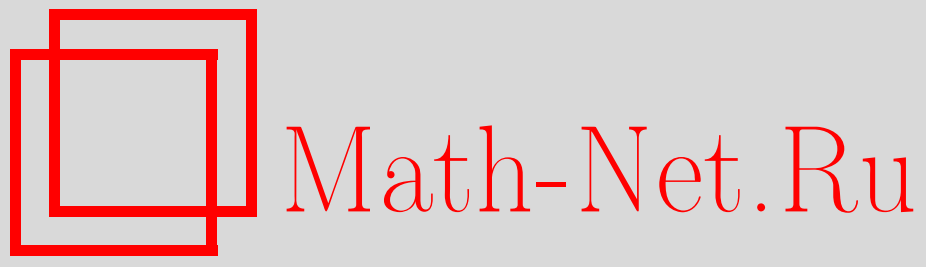

М. Л. Горбачук, Ю. Г. Мокроусов, О плотности подпространств аналитических векторов замкнутого оператора в банаховом пространстве, Функи. анализ и его прил., 2001, том 35, выпуск 1, 77-80

DOI: https://doi.org/10.4213/faa234

Использование Общероссийского математического портала MathNet.Ru подразумевает, что вы прочитали и согласны с пользовательским соглашением

http://www.mathnet.ru/rus/agreement

Параметры загрузки:

IP : 34.239 .49 .27

26 апреля 2023 г., 13:32:55

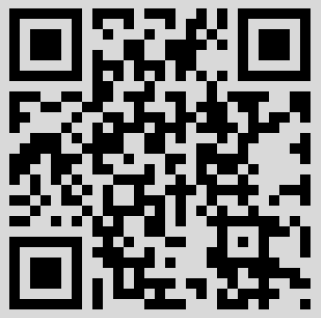


сферы относительно одной из точек (пример 1 выше), а также вращения, переводящие эту точку на любое расстояние от исходной (пример 2). Поэтому образ есть вся группа $S O(5)$. Ядро состоит из двух матриц, \pm 1 , так как любая другая матрица из $\mathbb{H} U(2)$ сдвигает некоторые точки $\mathbb{H} P^{1}$. Теорема доказана (при помощи кватернионного аналога тангенса половинного угла, роль которого играет кватернион $q$; соответствующая ему точка $s$ сферы $S^{4}$ играет роль самого исходного угла). Этот метод доставляет также много рациональных точек на сфере $S^{7}: x_{1}=\left(1-q^{2}\right)\left(1+q^{2}\right)^{-1}, x_{2}=2 q\left(1+q^{2}\right)^{-1}$.

ЗАмечАниЕ. При $n>2$ гиперунитарная группа $\mathbb{H} U(n)$ не спинорная: она имеет тип $C_{n}$, а не $B_{n}$.

\title{
ЛИТЕРАТУРА
}

1. Арнольд В. И. Функц. анализ и его прил., 1, вып. 1, 1-14 (1967). 2. Арнольд В. И. Труды МИАН, 224, 56-67 (1999). 3. Арнольд В. И. Функц. анализ и его прил., 34, вып. 3, 63-65 (2000).

Математический институт им. В. А. Стеклова, CEREMADE, Universitè Paris-Dauphine 31 октября 2000 г.

УДК $517.98+517.5$

\section{О плотности подпространств аналитических векторов замкнутого оператора в банаховом пространстве*}

\author{
(c) 2001. М. Л. ГОРБАЧУк, Ю. Г. МОКроУсов
}

1. Пусть $A$ - замкнутый оператор с плотной областью определения $\mathscr{D}(A)$ в комплексном банаховом пространстве $\mathfrak{B}$ с нормой $\|\cdot\|$. Обозначим через $C^{\infty}(A)$ пространство бесконечно дифференцируемых векторов оператора $A$ :

$$
C^{\infty}(A)=\bigcap_{n \in \mathbb{N}_{0}} \mathscr{D}\left(A^{n}\right) \quad\left(\mathbb{N}_{0}=0,1,2, \ldots\right) .
$$

Для числа $\beta \geqslant 0$ положим

$$
\begin{gathered}
\mathfrak{G}_{\{\beta\}}(A)=\left\{x \in C^{\infty}(A) \mid \exists \alpha>0 \exists c>0 \forall k \in \mathbb{N}_{0}\left\|A^{k} x\right\| \leqslant c \alpha^{k} k^{k \beta}\right\}, \\
\mathfrak{G}_{(\beta)}(A)=\left\{x \in C^{\infty}(A) \mid \forall \alpha>0 \exists c>0 \forall k \in \mathbb{N}_{0}\left\|A^{k} x\right\| \leqslant c \alpha^{k} k^{k \beta}\right\} .
\end{gathered}
$$

В первом из определенных выше множеств постоянные $\alpha$ и $c$ зависят от $x$, а во втором константа $c$ зависит также от $\alpha$. Линейные пространства $\mathfrak{G}_{\{\beta\}}(A)$ и $\mathfrak{G}_{(\beta)}(A)$ снабжаются топологиями соответственно индуктивного и проективного пределов банаховых пространств

$$
\begin{gathered}
\mathfrak{G}_{\beta}^{\alpha}(A)=\left\{x \in C^{\infty}(A) \mid \exists c>0 \forall k \in \mathbb{N}_{0}\left\|A^{k} x\right\| \leqslant c \alpha^{k} k^{k \beta}\right\}, \quad \alpha>0, \\
\|x\|_{\mathfrak{G}_{\beta}^{\alpha}(A)}=\sup _{k \in \mathbb{N}_{0}} \frac{\left\|A^{k} x\right\|}{\alpha^{k} k^{k \beta}} .
\end{gathered}
$$

*Работа поддержана Совместным грантом CRDF и правительства Украины UM 1-2090 и Государственным фондом фундаментальных исследований Украины (проект 1.4162). 
Таким образом,

$$
\mathfrak{G}_{\{\beta\}}(A)=\underset{\alpha \rightarrow \infty}{\operatorname{ind} \lim _{\beta}} \mathfrak{G}_{\beta}^{\alpha}(A), \quad \mathfrak{G}_{(\beta)}(A)=\underset{\alpha \rightarrow 0}{\operatorname{proj} \lim } \mathfrak{G}_{\beta}^{\alpha}(A) .
$$

Заметим, что для любых $\lambda, \mu \in \mathbb{C}$

$$
\mathfrak{G}_{\{\beta\}}(A)=\mathfrak{G}_{\{\beta\}}(\lambda A+\mu I) \text { при } \beta \geqslant 0, \quad \mathfrak{G}_{(\beta)}(A)=\mathfrak{G}_{(\beta)}(\lambda A+\mu I) \text { при } \beta>0
$$

$(\mathbb{C}-$ множество всех комплексных чисел, $I-$ тождественный оператор в $\mathfrak{B})$. Пространства $\mathfrak{G}_{\{1\}}(A), \mathfrak{G}_{(1)}(A)$ и $\mathfrak{G}_{\{0\}}(A)$ называются пространствами соответственно аналитических векторов [1], целых векторов [2] и целых векторов экспоненциального типа [3] оператора $A$.

В конкретном случае, когда $\mathfrak{B}=C[a, b],-\infty<a<b<\infty, A=d / d x$, $\mathscr{D}(A)=C^{1}[a, b]$, пространство $C^{\infty}(A)$ совпадает с множеством $C^{\infty}([a, b])$ всех бесконечно дифференцируемых на $[a, b]$ функций, а $\mathfrak{G}_{\{1\}}(A), \mathfrak{G}_{(1)}(A)$ и $\mathfrak{G}_{\{0\}}(A)$ суть не что иное, как пространства соответственно всех аналитических на $[a, b]$ функций, всех целых функций и всех целых функций экспоненциального типа. Пространство $\mathfrak{G}_{\{\beta\}}(A)\left(\mathfrak{G}_{(\beta)}(A)\right)$ с $\beta>1$ известно как класс Жевре типа Румье (Берлинга). Если же $\mathfrak{B}=L_{2}(\mathbb{R})(\mathbb{R}-$ множество всех действительных чисел) и $A-$ замыкание оператора $-d^{2} / d x^{2}+x^{2}$, заданного на множестве $C_{0}^{\infty}(\mathbb{R})$ всех финитных бесконечно дифференцируемых на $\mathbb{R}$ функций, то, как показано в [4], $C^{\infty}(A)=S$, а $\mathfrak{G}_{\{\beta\}}(A)=S_{\beta / 2}^{\beta / 2}, \beta \geqslant 1$, где $S-$ пространство Шварца, а $S_{\beta / 2}^{\beta / 2}-$ соответствующее пространство Гельфанда-Шилова из [5].

В приведенных примерах все рассмотренные пространства плотны в $\mathfrak{B}$. В общем случае это не так. Поэтому возникает вопрос, при каких условиях на оператор $A$ и число $\beta$ имеет место равенство $\overline{\mathfrak{G}_{(\beta)}(A)}=\mathfrak{B}$ или $\overline{\mathfrak{G}_{\{\beta\}}(A)}=\mathfrak{B}$. Этот вопрос интересовал многих математиков в различных более частных ситуациях (по этому поводу см. $[6,7]$ ). В [8] для $\beta>1$ сформулированная задача решена в терминах расположения спектра оператора $A$ и оценки роста его резольвенты при подходе к спектру. Нас будет интересовать случай $0 \leqslant$ $\beta \leqslant 1$. Следует отметить особую важность этого случая в теории приближения функций (см. [9]) и вопросах корректной разрешимости задачи Коши для дифференциально-операторных уравнений в различных пространствах, состоящих из аналитических функций [10].

2. Для чисел $c>0, R>0, \gamma \in[0,1)$ положим

$$
K_{R}=\{\lambda \in \mathbb{C}:|\lambda| \leqslant R\}, \quad \Pi_{\gamma, c}=\left\{\lambda \in \mathbb{C}:-c|\operatorname{Re} \lambda|^{\gamma} \leqslant \operatorname{Im} \lambda \leqslant c|\operatorname{Re} \lambda|^{\gamma}\right\} .
$$

Теорема 1. Пусть $A-$ замкнутый оператор в банаховом пространстве $\mathfrak{B}, \overline{\mathscr{D}(A)}=\mathfrak{B}$ и резольвента $R_{z}(A)=(A-z I)^{-1}$ существует в области $\mathbb{C} \backslash\left(K_{R} \cup \Pi_{\gamma, c}\right)$ при некоторых $c>0, R>0, \gamma \in[0,1)$ и там удовлетворяет оценке

$$
\left\|R_{z}(A)\right\| \leqslant M(1+|z|)^{N},
$$

где $M, N-$ положительные константы. Тогда для произвольного $\beta>0$

$$
\overline{\mathfrak{G}_{(\beta)}(A)}=\mathfrak{B} .
$$

Заметим, что в условиях этой теоремы тем более $\overline{\mathfrak{G}_{\{\beta\}}(A)}=\mathfrak{B}$. 
Главным моментом в доказательстве теоремы 1 является установление того факта, что при любом $\beta>0$ множество элементов вида

$$
\int_{\Gamma} h_{\varepsilon}(z) R_{z}(A) x d z, \quad \varepsilon \in(0, \infty), x \in C^{\infty}(A),
$$

принадлежит $\mathfrak{G}_{(\beta)}(A)$ и плотно в $\mathfrak{B}$. Здесь $h_{\varepsilon}(z)=h(\varepsilon z), h(z)-$ целая функция, удовлетворяющая неравенству

$$
|h(z)| \leqslant c e^{-a|\operatorname{Re} z|^{1 / \beta}+b|\operatorname{Im} z|^{1 / \beta}}
$$

с некоторыми положительными константами $c, a$ и $b$, а соответствующим образом выбранный контур $Г$ лежит в резольвентном множестве оператора $A$. Существование нетривиальной целой функции $h(z)$ со свойством (1) доказано в [5].

СледствиЕ 1. Если $A$ - генератор однопараметрической группь ограниченных линейных операторов в $\mathfrak{B}$ класса $C_{0}$, mо $\overline{\mathfrak{G}_{(\beta)}(A)}=\mathfrak{B}$ при любом $\beta>0$.

В самом деле, в этом случае оператор $i A$, как показано в [6], удовлетворяет условиям теоремы 1 с $\gamma=0, N=-1$.

Заметим, что теорема 1 , вообще говоря, не верна при $\beta=0$. Можно построить пример оператора, удовлетворяющего условиям этой теоремы и даже следствия 1 , для которого $\mathfrak{G}_{\{0\}}(A)=\{0\}$.

3. Напомним, что полугруппа линейных операторов $U(t), t \geqslant 0$, класса $C_{0}$ называется аналитической с углом $\pi / 2$, если она допускает аналитическое продолжение в полуплоскость $\operatorname{Re} z>0$, непрерывное в нуле по любому лучу, исходящему из начала координат и лежащему в этой полуплоскости. Известно [6], что спектр генератора $A$ такой полугруппы лежит в $(-\infty, \omega]$, где $\omega-$ некоторое действительное число. Положим

$$
M(s)=\sup _{|\operatorname{Im} \lambda| \geqslant s}\left\|R_{\lambda}(A)\right\|
$$

Теорема 2. (1) Пусть $A-$ генератор аналитической полугруппь $U(t), t \geqslant 0$, с углом $\pi / 2$. Тогда $\overline{\mathfrak{G}_{(\beta)}(A)}=\mathfrak{B}$ для любого $\beta>0$.

(2) Существуют аналитические полугруппь с углом $\pi / 2$, такие, что $\mathfrak{G}_{\{0\}}(A)=\{0\}$. Однако если аналитическая полугруппа $U(t), t \geqslant 0, c$ углом $\pi / 2$ такова, что

mo $\overline{\mathfrak{G}_{\{0\}}(A)}=\mathfrak{B}$.

$$
\int_{0}^{1} \ln \ln M(s) d s<\infty
$$

4. Пусть теперь $A$ - произвольный замкнутый оператор в $\mathfrak{B}$, для которого $\overline{\mathfrak{G}_{(\beta)}(A)}=\mathfrak{B}, 0 \leqslant \beta \leqslant 1$. Определим оператор-функцию $U(z), z \in \mathbb{C}$, формулой

$$
U(z) x=e^{z A} x=\sum_{k=0}^{\infty} \frac{z^{k} A^{k} x}{k !} .
$$

Нетрудно показать, что для каждого $x \in \mathfrak{G}_{(\beta)}(A), 0 \leqslant \beta \leqslant 1$, ряд в $(2)$ сходится в топологии пространства $\mathfrak{G}_{(\beta)}(A)$ равномерно по $z$ на любом компакте из $\mathbb{C}$. Он также сходится в $\mathfrak{G}_{\{\beta\}}(A)$, но уже при $\beta \in[0,1)$. 
Теорема 3. Oператор-функция $U(z), z \in \mathbb{C}$, определенная формулой (2), является аналитической группой в пространстве $\mathfrak{G}_{(\beta)}(A)$ при $0 \leqslant \beta \leqslant 1$ и в пространстве $\mathfrak{G}_{\{\beta\}}(A)$ при $0 \leqslant \beta<1$.

Если оператор $A$, фигурирующий в теореме 3 , является генератором полугруппы класса $C_{0}$ и $\overline{\mathfrak{G}_{(\beta)}(A)}=\mathfrak{B}, 0 \leqslant \beta \leqslant 1$, то, каков бы ни был сегмент $[0, T]$, $T>0$, любое решение уравнения

$$
\frac{d y(t)}{d t}=A y(t), \quad t \in[0, T],
$$

можно приблизить равномерно на этом промежутке решениями, являющимися целыми вектор-функциями. Последние же равномерно приближаются частными суммами ряда $(2)$.

\section{ЛИТЕРАТУРА}

1. Nelson E. Ann. of Math., 70, No. 3, 572-615 (1959). 2. Goodman R. Trans. Amer. Math. Soc., 143, No. 3, 55-76 (1969). 3. Радыно Я. В. Докл. АН БССР, 27, o. 9, 791-793 (1983). 4. Кашпировский А. И. Функц. анализ и его прил., 14, вып. 2, 60-61 (1980). 5. Гельфанд И. М., Шилов Г. Е. Пространства основных и обобщенных функций. Физматгиз, М., 1958. 6. Рид М., Саймон Б. Методы современной математической физики. Гармонический анализ. Самосопряженность. Мир, М., 1978. 7. Горбачук В. И., Князюк А. В. УМН, 44, o. 3, 55-91 (1989). 8. Beals R. J. Funct. Anal., 10, No. 3, 300-308 (1972). 9. Горбачук М. Л., Горбачук В. И. Укр. матем. ж., 47, o. 5, 616-628 (1995). 10. Горбачук М. Л. Математичні методи та фізико-механічні поля, 41, №. 2, 7-12 (1998).

Институт математики НАН Украины

Национальный технический университет Украины «КПИ»

Поступило в редакцию 6 августа 1999 г.

УДК 512.643.5

\section{Асимптотика наименьших собственных значений матриц типа Гильберта*}

(c) 2001. Г. А. КАляБин

1. Рассмотрим семейство матриц $H_{n}=H_{n}(\beta, \theta), \beta>0, \theta>0$, типа Гильберта

$$
H_{n}=\left\|h_{j, k}\right\|, \quad h_{j, k}=h_{j, k}(\beta, \theta):=\frac{\beta^{j+k}}{j+k+\theta}, \quad j, k \in\{0,1, \ldots n\},
$$

являющихся матрицами Грама системы степеней $\left\{1, x, \ldots, x^{n}\right\}$ на интервале $[0, \beta]$ с весом $w(x)=x^{\theta-1} \beta^{-\theta}$ (см. $\left.[1, \S 10.1]\right)$ и играющих важную роль в различных задачах аппроксимации и экстраполяции.

Целью настоящей работы является доказательство следующей асимптотической формулы для наименьших собственных значений матриц $H_{n}(\beta, \theta)$.

Теорема. При $n \rightarrow \infty$ имеет место предельное соотношение

$$
\lambda_{\min }\left(H_{n}(\beta, \theta)\right)=K \sqrt{n} \rho^{-4 n}(1+o(1)),
$$

* Работа была поддержана грантами Европейского сообщества (INTAS-881-94) и Российского фонда фундаментальных исследований (РФФИ-99-01-00868). 